\title{
Search for solid HDO in low-mass protostars
}

\author{
B. Parise ${ }^{1}$, T. Simon ${ }^{2}$, E. Caux ${ }^{1}$, E. Dartois ${ }^{3}$, C. Ceccarelli ${ }^{4}$, J. Rayner ${ }^{2}$, and A. G. G. M. Tielens ${ }^{5}$ \\ 1 CESR CNRS-UPS, BP 4346, 31028 Toulouse Cedex 04, France \\ 2 Institute for Astronomy, 2680 Woodlawn Drive, Honolulu, HI 96822, USA \\ ${ }^{3}$ IAS-CNRS, Bât. 121, Université Paris Sud, 91405 Orsay Cedex, France \\ ${ }^{4}$ Laboratoire d'Astrophysique, Observatoire de Grenoble, BP 53, 38041 Grenoble Cedex 09, France \\ 5 SRON, PO Box 800, 9700 AV Groningen, The Netherlands
}

Received 21 May 2003 / Accepted 11 August 2003

\begin{abstract}
We present ground-based 2.1 to $4.2 \mu \mathrm{m}$ observations of four low-mass protostars. We searched for the $4.1 \mu \mathrm{m}$ OD stretch band, characteristic of solid HDO in grain mantles. We did not detect solid HDO in any of the four sources, but we derive $3 \sigma$ upper limits from $0.5 \%$ to $2 \%$ for the $\mathrm{HDO} / \mathrm{H}_{2} \mathrm{O}$ ratio depending on the source. These ratios provide strong constraints to solid-state deuteration models when compared to deuterium fractionation values observed in the gas phase. We discuss various scenarios that could lead to such a low water deuteration compared to the high formaldehyde and methanol deuteration observed in the gas-phase.
\end{abstract}

Key words. astrochemistry - ISM: abundances, molecules, lines and bands - stars: formation - individual objects: NGC 1333 SVS12, SVS13, L1489 IRS, TMR1

\section{Introduction}

Large deuteration fractionations - ratio of deuterated over normal isotope - are a common characteristic of dark, dense molecular clouds, with abundances of deuterated species some $0.01-0.1$ of the normal isotopomer (e.g. Turner 2001). This deuteration is thought to reflect the small zero point vibrational energy differences between a deuterated species and its fully hydrogenated counterpart, which drives the chemistry towards deuterated species at low temperatures (10 K; Watson 1973). In view of their elevated temperatures $(\geq 100 \mathrm{~K})$, the warm gas around protostars shows unexpected high abundances of deuterated species. Indeed, fractionations of $10^{-3}$ to more than 0.1 have previously been observed. Low-mass protostars seem to show even greater fractionations than high-mass protostars. Indeed, observations of the low-mass protostar IRAS 16293-2422 showed that $\mathrm{D}_{2} \mathrm{CO} / \mathrm{H}_{2} \mathrm{CO} \sim 10 \%$ (Ceccarelli et al. 1998), a fractionation about 25 times larger than in the Hot Core in Orion (Turner 1990). Similarly large amounts of doublydeuterated formaldehyde have subsequently been observed towards a sample of low-mass protostars (Loinard et al. 2002). Singly- and doubly-deuterated methanol were detected towards IRAS 16293-2422 (Parise et al. 2002), showing fractionation ratios of $0.9\left(\mathrm{CH}_{2} \mathrm{DOH}\right)$ and $0.2\left(\mathrm{CHD}_{2} \mathrm{OH}\right)$. Deuterated methanol was also observed towards a sample of other lowmass protostars (Parise et al., in prep.), confirming the huge deuteration in these objects, whereas the fractionation ratio

Send offprint requests to: B. Parise, e-mail: Parise@cesr . fr
$\mathrm{CH}_{2} \mathrm{DOH} / \mathrm{CH}_{3} \mathrm{OH}$ is only 0.04 in the Orion high-mass starforming region (Jacq et al. 1993).

Because the high temperatures of the gas around protostars prevent significant deuterium fractionation, the observed high deuterations are thought to reflect a previous cold phase. Molecules that formed during the dark cloud phase - either in the gas phase or on the grain surfaces - are believed to be stored in an ice mantle on dust grains, which evaporates once the YSO heats its environment above the ice sublimation temperature (Ceccarelli et al. 2001). Presumably the current gas phase has not had time to return to equilibrium, which takes some $3 \times 10^{4}$ years (Charnley et al. 1992; Caselli et al. 1993; Charnley et al. 1997).

A previous detection of solid-state HDO in the high-mass luminous W33A and NGC 7538 IRS9 protostars has been claimed by Teixeira et al. (1999). However, recent analysis of the same data seems to challenge this detection, giving an upper limit of $10^{-2}$ for solid $\mathrm{HDO} / \mathrm{H}_{2} \mathrm{O}$ in NGC 7538 IRS9 (Dartois et al. 2003). Subsequent VLT observations of W33A also give an upper limit for the deuterium fractionation of $\sim 10^{-2}$ (Dartois et al. 2003), a value still consistent with the typical deuteration observed for the gas phase of hot cores around high mass protostars. The search for HDO in highmass protostars was thus thought to be inconclusive largely because the expected fractionation was very small. Because low-mass YSOs show gas-phase deuterium fractionations almost 100 times higher in their hot cores, the solid HDO feature is expected to be much stronger, and motivated this study. Presently published grain chemistry models predict an HDO 
fractionation of about $40 \%$ (i.e. almost all the deuterium would be locked in HDO) if deuterated water forms on the grain surfaces at the same time as formaldehyde and methanol (Caselli et al. 2002; Stantcheva \& Herbst 2003).

In this paper, we present a search for the HDO stretch-band at $4.1 \mu \mathrm{m}$ towards four low-mass protostars. In Sect. 2 we describe the observations, and derive the $\mathrm{H}_{2} \mathrm{O}$ ice column density and upper limits for HDO column densities in Sect. 3. We discuss the derived fractionation ratios, comparing to available gas-phase observations (for SVS12 and SVS13) in Sect. 4, and conclude in Sect. 5.

\section{Observations}

\subsection{Source selection}

As noted in the Introduction, the previous searches for solid HDO have been carried out towards high-mass protostars, with very modest success. In this study we focused on low-mass protostars, where gas-phase deuteration has been observed to be much higher than in high-mass protostars.

The sources of the present study were selected to be bright enough at NIR wavelengths, so that high enough $\mathrm{S} / \mathrm{N}$ can be obtained to detect weak absorption features against the continuum, and to have large $J-K$ colour index, which would indicate a high extinction.

The first criterion, by definition, excludes the most embedded low-mass protostars, the Class 0 sources, because the NIR continuum is too faint, leaving the more evolved Class I sources. Even though not totally conclusive, the study of Loinard et al. (2002) on a sample of four Class I sources seems to indicate that also in those sources the molecular deuteration, and specifically the $\mathrm{D}_{2} \mathrm{CO} / \mathrm{H}_{2} \mathrm{CO}$ ratio, remains relatively large and comparable to that found in Class 0 sources. Encouraged by this, we selected the four sources in Table 1. Two of our sources were observed by Loinard et al. (2002). In the following we give a brief description of each selected source.

NGC 1333 SVS12, also called IRAS 6, is probably a Class I source of $\sim 28 L_{\odot}$ in the IRAS bands (Jennings et al. 1987) assuming a distance of $350 \mathrm{pc}$. New distance estimates of the NGC 1333 complex tend to put it closer at about $220 \mathrm{pc}$ (Cernis 1990), which would bring the SVS12 luminosity to $\sim 10 L_{\odot}$. The infrared source is close to the Herbig Haro object HH12, although SVS12 probably is not the exciting source of the HH12 flow. Millimetre and submillimetre maps resolved the source in possibly three components (Sandell \& Knee 2001). A detailed CO 2-1 map of the region did not detect any outflow emanating from this source (Knee \& Sandell 2000) so it may be a relatively evolved source indeed, and possibly a background Class II source. In this case, we may be observing the deuteration in the cloud ices rather than in the circumstellar material. Loinard et al. (2002) detected abundant doubly deuterated formaldehyde, $\mathrm{D}_{2} \mathrm{CO} / \mathrm{H}_{2} \mathrm{CO} \sim 5 \%$.

NGC 1333 SVS13, also called IRAS 03259+3105 (Jennings et al. 1987), is the best studied source of the region, thanks to its well collimated flow of $\mathrm{HH}$ objects, the HH7-11 complex. It is a variable object and one of the brightest NIR sources of the region, having an average bolometric luminosity of $115 L_{\odot}$ (Molinari et al. 1993; $45 L_{\odot}$ if the distance is $220 \mathrm{pc}$ ), and it is very probably a multiple system (Lefloch et al. 1998a,b; Bachiller et al. 1998; Looney et al. 2000). Also in this source Loinard et al. (2002) detected abundant doubly deuterated formaldehyde, $\mathrm{D}_{2} \mathrm{CO} / \mathrm{H}_{2} \mathrm{CO} \sim 4 \%$.

L1489 IRS (IRAS 04016+2610) is a low-luminosity source $\left(L_{\mathrm{bol}}=3.7 L_{\odot}\right)$, which acquired recent attention because it is suspected to be one of the rare cases of a source in transition from Class I to Class II (Hogerheijde \& Sandell 2000; Hogerheijde 2001). It appears to be surrounded by a relatively massive and young disk (Boogert et al. 2002a), whereas the envelope seems to be largely swept out.

TMR1 (IRAS 04361+2547) is a prototypical Class I, lowluminosity source $\left(L_{\text {bol }}=2.9 L_{\odot}\right.$ : Hogerheijde et al. 1998). Both L1489 IRS and TMR1 belong to the Taurus complex at a distance of $140 \mathrm{pc}$.

\subsection{Observations}

Using the SpeX instrument (Rayner et al. 2003) on the IRTF telescope on Mauna Kea (Hawaii), we observed the four lowmass class I protostars listed in Table 1. The observations were performed in December 2002 under conditions of good seeing $\left(0.6^{\prime \prime}\right.$ at $\mathrm{K}$, on average). We used the $2.1-5.0 \mu \mathrm{m}$ crossdispersed mode, which acquires the full spectrum between 2.1 and $5.0 \mu \mathrm{m}$ simultaneously. We used the $0.3^{\prime \prime}$ slit, which provides a spectral resolution of 2500 , for the first three objects, and the $0.5^{\prime \prime}$ slit $(R=1500)$ for TMR1. Standard stars were chosen from the IRTF A0V-star database to be as bright and as close to the studied object as possible, and are listed in Table 1. The airmass difference between the object and the associated standard was always less than 0.1 .

The observations were reduced using the Spextool software (Vacca et al. 2003; Cushing et al. in prep.), which is available on the IRTF SpeX webpage (http://irtfweb. ifa.hawaii.edu/Facility/spex/). The full spectra for the four protostars, smoothed to a resolution of $\sim 10 \mathrm{~cm}^{-1}$, are presented in Fig. 1.

The HDO absorption feature was not detected in any of the four sources. We derive upper limits for the $\mathrm{HDO} / \mathrm{H}_{2} \mathrm{O}$ ratio in next section. The spectra reveal two hydrogen lines at $2468 \mathrm{~cm}^{-1}(4.05 \mu \mathrm{m})$ and $2673 \mathrm{~cm}^{-1}(3.74 \mu \mathrm{m})$, as well as CO emission lines (SVS13, Carr et al. 1992). The deep absorption feature at $3300 \mathrm{~cm}^{-1}(3.03 \mu \mathrm{m})$ is the $\mathrm{OH}$ stretch band. On its right wing, the so-called " $3.47 \mu \mathrm{m}$ feature" $\left(2882 \mathrm{~cm}^{-1}\right)$ is visible.

\section{Water features}

\subsection{The $\mathrm{OH}$ stretch band}

All four spectra show a prominent $\mathrm{OH}$ absorption feature at $3300 \mathrm{~cm}^{-1}(3.03 \mu \mathrm{m})$. The abundance of solid hydrogenated water can be derived from this $\mathrm{OH}$ stretch band. We first define the continuum $I_{\text {cont }}$ around the $\mathrm{OH}$ feature.

We can then plot the optical depth $\tau$ defined by:

$I / I_{\text {cont }}=\mathrm{e}^{-\tau}$ 
Table 1. Observed sources, coordinates, NIR magnitude, $J-K$ colour index (indicative of extinction, cf. text), $K$ magnitude and observation parameters.

\begin{tabular}{|c|c|c|c|c|c|c|c|c|c|}
\hline Source & $\alpha(2000.0)$ & $\delta(2000.0)$ & $m_{L}$ & $\begin{array}{l}L \text {-band } \\
\text { flux (Jy) }\end{array}$ & $J-K$ & $\bar{K}$ & Slit / Resolution & $\begin{array}{l}\text { On-source } \\
\text { int time }\end{array}$ & Standard \\
\hline NGC 1333/SVS12 & $03^{\mathrm{h}} 29^{\prime} 01.4^{\prime \prime}$ & $31^{\circ} 20^{\prime} 21^{\prime \prime}$ & & & 4.24 & 10.61 & $0.3^{\prime \prime} / 2500$ & $20 \mathrm{~min}$ & HD 20995 \\
\hline NGC 1333/SVS13 & $03^{\mathrm{h}} 29^{\prime} 03.7^{\prime \prime}$ & $31^{\circ} 16^{\prime} 03^{\prime \prime}$ & 5.4 & 1.99 & 3.74 & 8.25 & $0.3^{\prime \prime} / 2500$ & $15 \mathrm{~min}$ & HD 20995 \\
\hline L1489 IRS & $04^{\mathrm{h}} 04^{\prime} 42.9^{\prime \prime}$ & $26^{\circ} 18^{\prime} 56^{\prime \prime}$ & 6.8 & 0.55 & & 9.3 & $0.3^{\prime \prime} / 2500$ & $20 \mathrm{~min}$ & HD 23258 \\
\hline TMR1 & $04^{\mathrm{h}} 39^{\prime} 13.9^{\prime \prime}$ & $25^{\circ} 53^{\prime} 21^{\prime \prime}$ & 8.9 & 0.08 & 5.55 & 10.54 & $0.5^{\prime \prime} / 1500$ & $53 \mathrm{~min}$ & HD 31592 \\
\hline
\end{tabular}
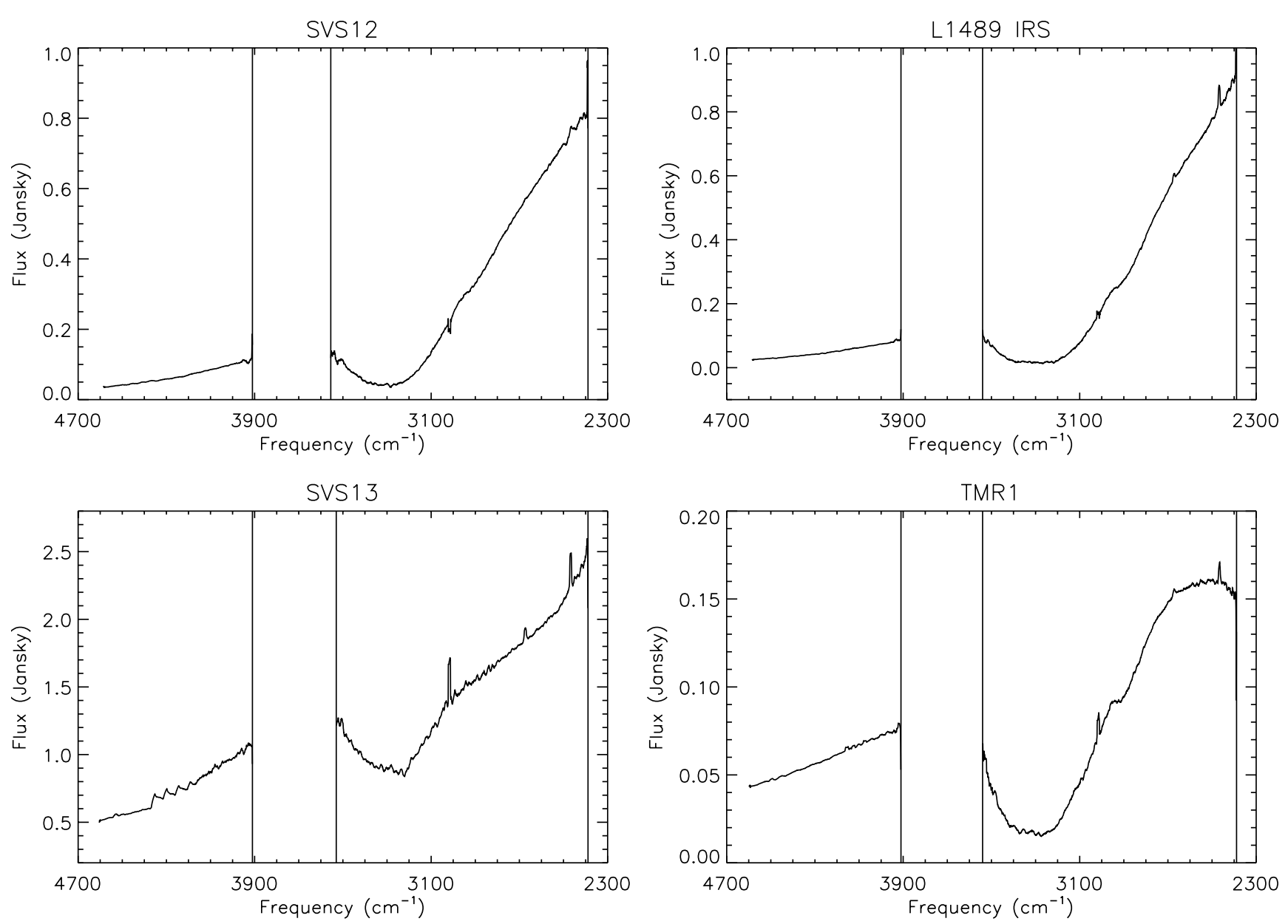

Fig. 1. Spectra of the four protostars, smoothed to $10 \mathrm{~cm}^{-1}$ resolution. The vertical lines delineate the region of strong atmospheric absorption features.

The column density of $\mathrm{H}_{2} \mathrm{O}$ molecules can be derived from the optical depth with the following relation:

$N_{\mathrm{H}_{2} \mathrm{O}}=\frac{\int \tau \mathrm{d} v}{A_{\mathrm{H}_{2} \mathrm{O}}}$,

where the band strength $A_{\mathrm{H}_{2} \mathrm{O}}=2 \times 10^{-16} \mathrm{~cm} /$ molecule (Dartois 1998) and $\int \tau \mathrm{d} v$ is expressed in $\mathrm{cm}^{-1}$.

\subsubsection{SVS12, L1489 IRS and TMR1}

For these three sources, we defined the local continuum around the $\mathrm{OH}$ feature by fitting a second order polynomial to the frequency ranges [2400-2500, 2700] and [4000, 4400$4600 \mathrm{~cm}^{-1}$. These frequency ranges were chosen to avoid the region around the $2882 \mathrm{~cm}^{-1}(3.47 \mu \mathrm{m})$ feature in the continuum fit. An example of the derived continuum is shown in Fig. 2.

The water feature for the three sources shows no structure and is thus attributable to amorphous ice. We integrated the contribution of the band between 3000 and $3555 \mathrm{~cm}^{-1}$. This integrated optical depth corresponds to a lower limit on the $\mathrm{H}_{2} \mathrm{O}$ column density. The $\mathrm{OH}$ bands for these three sources are plot in an optical depth scale in Fig. 3.

The optical depth for the $3300 \mathrm{~cm}^{-1}$ feature for each source and the derived $\mathrm{H}_{2} \mathrm{O}$ column density are summarized in Table 2. Our optical depth for L1489 IRS is consistent with the observation at low resolution $(R=150)$ by Sato et al. (1990, $\tau=2.9 \pm 0.2$ ), but we find a lower (2.0) optical depth for 


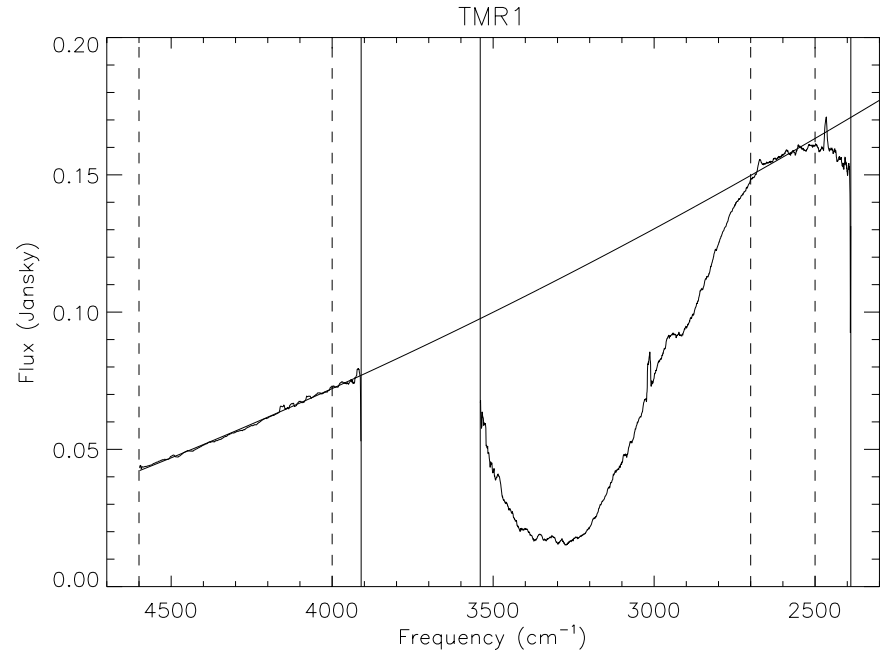

Fig. 2. Example of determination of the continuum for the study of the $3.0 \mu \mathrm{m}$ feature. The dotted lines show the frequency ranges used to fit the continuum.

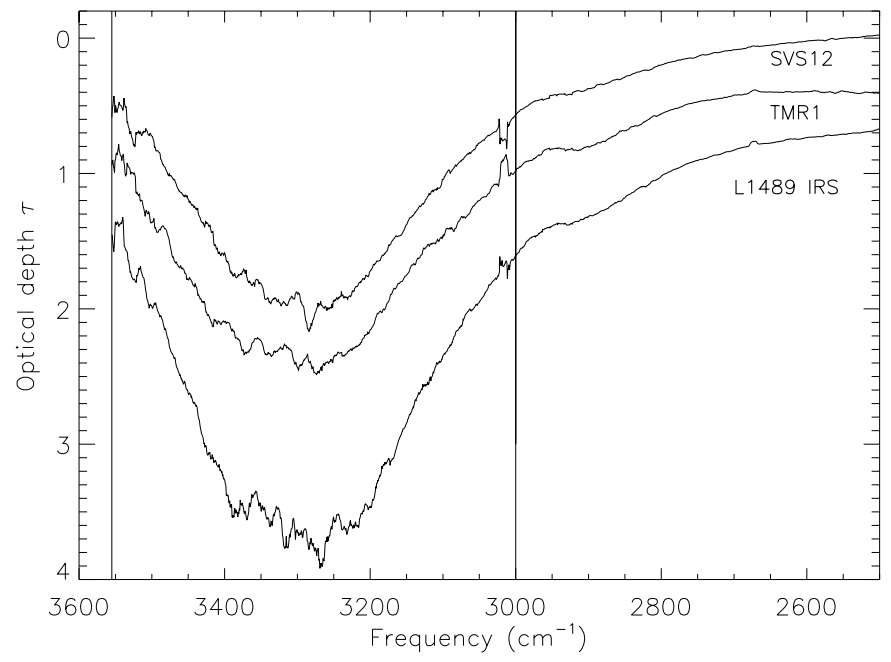

Fig. 3. OH stretch band for SVS12, L1489 IRS and TMR1. For clarity, the plots for TMR1 and L1489 IRS have been offset by 0.4 and 0.7 respectively. The vertical lines delineate the band where the integrated optical depth was computed.

$\operatorname{SVS12}(\tau=2.9 \pm 0.2$, Sato et al. 1990). This inconsistency certainly arises from the continuum determination, as Sato et al. only had observations between $2.4 \mu \mathrm{m}\left(4167 \mathrm{~cm}^{-1}\right)$ and $3.8 \mu \mathrm{m}$ $\left(2632 \mathrm{~cm}^{-1}\right)$.

\subsubsection{SVS13}

SVS13 appears to be a more unusual source than the other three. First of all, its $\mathrm{OH}$ stretch band has a non-Gaussian shape, which suggests that the ice is in a crystalline state. Second, the spectrum is characterized by a strange inflexion in the frequency range $[3900,4700]$ as well as $[2400,2600] \mathrm{cm}^{-1}$. The inflexion in the $[2400,2600] \mathrm{cm}^{-1}$ range cannot come from the standard star, as we used the same standard object for SVS12, which shows no inflexion. Moreover, ISO-SWS observations at low resolution of this source (ISO archive
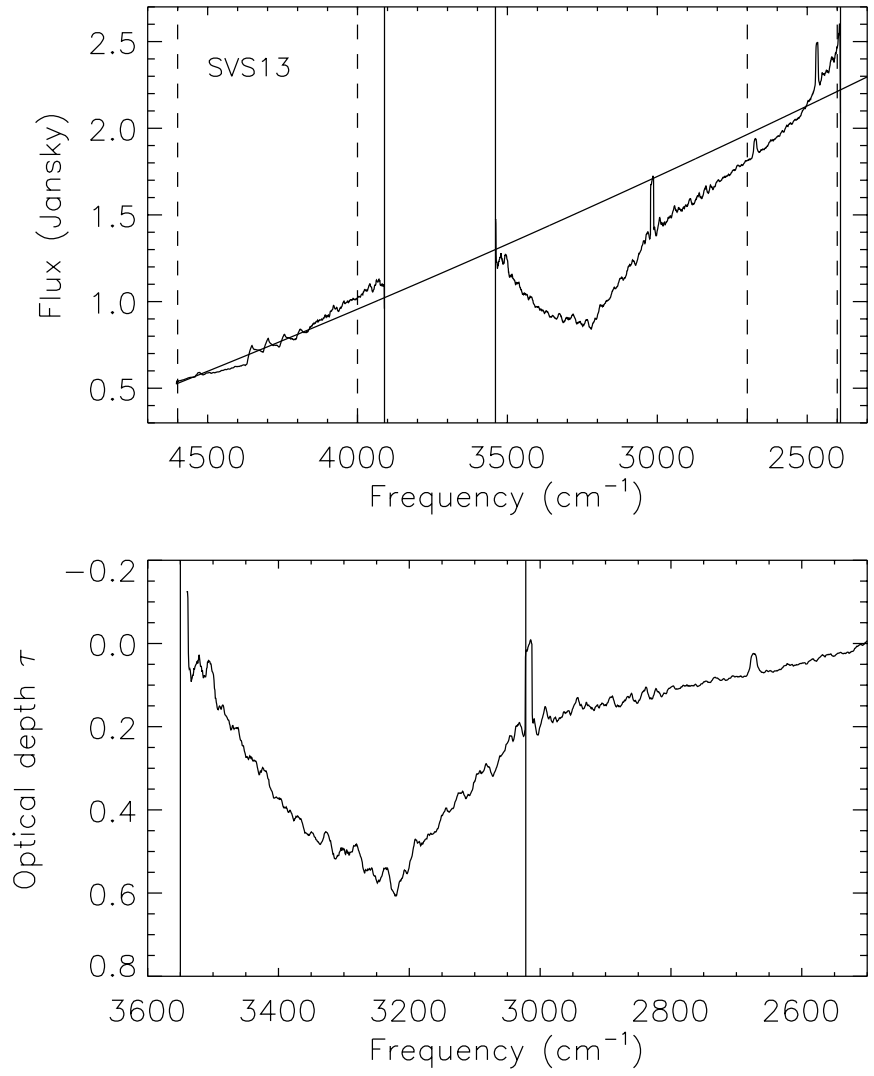

Fig. 4. Example of determination of the continuum for the study of the $3.0 \mu \mathrm{m}$ feature. The dotted lines show the frequency ranges used to fit the continuum.

tdt 65201959) have the same inflexion. This strange behaviour of the spectrum makes it difficult to define the local continuum.

We defined several different continua, by fitting first order polynomials on different anchoring frequency ranges. An example is shown in Fig. 4. In all cases, the optical depth plots have a broad absorption on the right side of the $\mathrm{OH}$ feature. This absorption could be due to scattering in the $\mathrm{OH}$ wing (Dartois et al. 2002). The integrated optical depth varies from 190 to $220 \mathrm{~cm}^{-1}$. We chose the smallest value for a lower limit on the $\mathrm{H}_{2} \mathrm{O}$ column density.

\subsection{Upper limits on the HDO abundance}

To determine upper limits on the HDO abundances, we first subtracted the two hydrogen lines at 2468 and $2673 \mathrm{~cm}^{-1}$. To do so, we fitted a first or second order polynomial in the [2400, $2800 \mathrm{~cm}^{-1}$ frequency range, ignoring a small frequency range around each of the two hydrogen lines (horizontal marks in Fig. 5). Continuum fits are plotted for each source in Fig. 5. The hydrogen lines were subtracted from the data by replacing the data in the associated frequency ranges by the interpolated continuum.

We then defined a local continuum around the expected $2457 \mathrm{~cm}^{-1}(4.07 \mu \mathrm{m})$ OD stretch band by fitting a first or second order polynomial in the [2410, 2440] and $[2500,2800] \mathrm{cm}^{-1}$ frequency range.

An upper limit on the HDO column density was derived taking into account that the expected feature is rather broad 
Table 2. $\mathrm{OH}$ and OD stretch band characteristics. Upper limits for HDO parameters are 3- $\sigma$. (a) In the amorphous case, (b) in the crystalline case (see text).

\begin{tabular}{cccccccc}
\hline \hline Source & \multicolumn{3}{c}{$\begin{array}{c}\int \tau_{\mathrm{OH}} \mathrm{d} v \\
\left(\mathrm{~cm}^{-1}\right)\end{array}$} & $\begin{array}{c}N\left(\mathrm{H}_{2} \mathrm{O}\right) \\
\left(10^{18} \mathrm{~cm}^{-2}\right)\end{array}$ & $\begin{array}{c}\tau_{\mathrm{OD}} \\
\left(\mathrm{cm}^{-1}\right)\end{array}$ & $\begin{array}{c}N(\mathrm{HDO}) \\
\left(10^{16} \mathrm{~cm}^{-2}\right)\end{array}$ & $\mathrm{HDO} / \mathrm{H}_{2} \mathrm{O}$ \\
\hline NGC 1333/SVS12 & 2.0 & $\geq 750$ & $\geq 3.8$ & $\leq 0.005$ & $\leq 0.63$ & $\leq 1.8$ & $\leq 0.5 \%$ \\
NGC 1333/SVS13(a) & 0.55 & $\geq 190$ & $\geq 1.0$ & $\leq 0.016$ & $\leq 2.0$ & $\leq 5.6$ & $\leq 5.9 \%$ \\
NGC 1333/SVS13(b) & & & & $\leq 0.022$ & $\leq 0.58$ & $\leq 1.6$ & $\leq 1.7 \%$ \\
L1489 IRS & 3.0 & $\geq 1160$ & $\geq 5.8$ & $\leq 0.013$ & $\leq 1.6$ & $\leq 4.4$ & $\leq 0.8 \%$ \\
TMR1 & 2.0 & $\geq 770$ & $\geq 3.9$ & $\leq 0.013$ & $\leq 1.6$ & $\leq 4.4$ & $\leq 1.1 \%$ \\
\hline
\end{tabular}
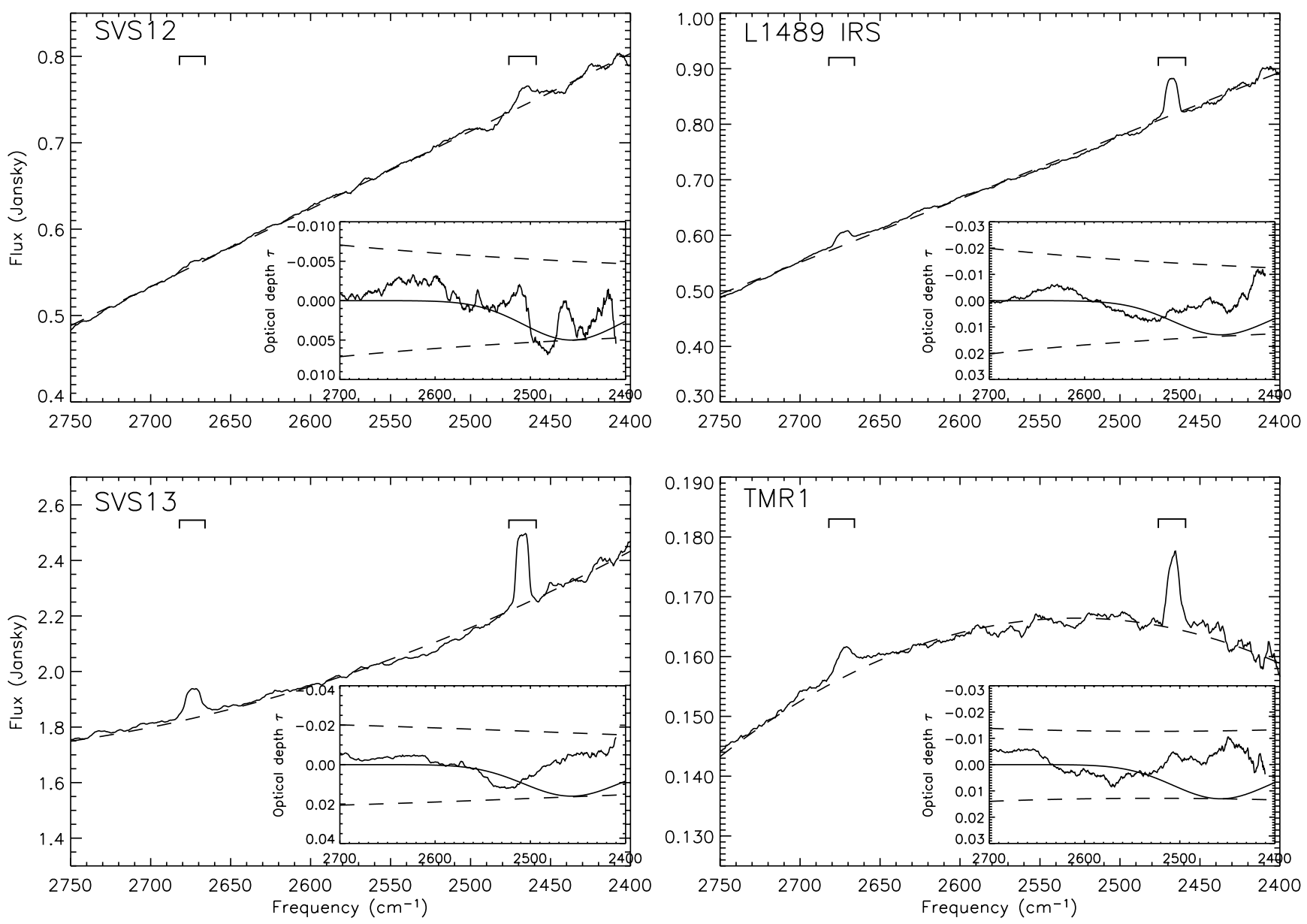

Fig. 5. OD stretch band for the four protostars. The horizontal bars show the frequency ranges ignored in the continuum fit. The continuum is drawn as a dashed line. In insets, the data are plotted on an optical depth scale versus frequency, after smoothing to $30 \mathrm{~cm}^{-1}$ resolution. The dashed lines are the $3 \sigma$ limits on the flux, transposed on an optical depth scale. The Gaussian shows the maximum amorphous HDO absorption.

$\left(120 \mathrm{~cm}^{-1}\right)$. The data were smoothed accordingly to a resolution of $30 \mathrm{~cm}^{-1}$. The upper limit on the HDO column density was then derived by estimating the biggest Gaussian feature fitting in a $3 \sigma$-wide band around the polynomial fit.

The Gaussian, centered on $2457 \mathrm{~cm}^{-1}$, was chosen to have a fixed FWHM of $120 \mathrm{~cm}^{-1}(0.2 \mu \mathrm{m})$, as measured in the laboratory for amorphous HDO (Dartois et al. 2003). The extreme Gaussians for each source are plotted on an optical depth scale in Fig. 5 (see insets). The dashed lines represent the $\pm 3 \sigma$ limits associated with the continuum determination at $30 \mathrm{~cm}^{-1}$ resolution.

The upper limit on the HDO column density is then derived from the relation:

$N_{\mathrm{HDO}} \leq \frac{\int \tau \mathrm{d} v}{A_{\mathrm{OD}}}$

where $\int \tau \mathrm{d} v$ is the area of the extreme Gaussian, and $A_{\mathrm{OD}}$ was taken to be $3.6 \times 10^{-17}$ for a conservative upper limit estimate $\left(A_{\mathrm{OD}}=(4.3 \pm 0.7) \times 10^{-17} \mathrm{~cm} /\right.$ molecule, Dartois et al. 2003) . 
For SVS13, we estimated an upper limit on the HDO column density for the case of crystalline ice. The Gaussian was then centered on $2427 \mathrm{~cm}^{-1}(4.12 \mu \mathrm{m})$, with a FWHM of $30 \mathrm{~cm}^{-1}(0.05 \mu \mathrm{m})$ following laboratory experiments (Dartois et al. 2003). In that case, the data were smoothed to $10 \mathrm{~cm}^{-1}$ resolution, the results for which are plotted in Fig. 6 .

The $3 \sigma$ upper limits for the HDO column densities and associated $\mathrm{HDO} / \mathrm{H}_{2} \mathrm{O}$ ratios are listed in Table 2.

\section{Discussion}

The observations presented here represent the first attempt to study the solid-phase deuteration in low-mass protostars, namely sources with luminosities of a few tens of solar luminosities. Previous studies focused on sources whose luminosity is larger than about $200 L_{\odot}$ (e.g. Dartois et al. 2003), and where the deuteration of gas phase molecules is relatively low. Specifically, the gas-phase $\mathrm{D}_{2} \mathrm{CO} / \mathrm{H}_{2} \mathrm{CO}$ ratio is $\leq 0.4 \%$ in high-mass protostars, whereas it is $\geq 4 \%$ in low-mass protostars (Loinard et al. 2002). For this reason, the present observations represent the most stringent constraint and a crucial test on the relation between the solid $\mathrm{H}_{2} \mathrm{O}$ deuteration and the large deuteration observed in the minor gas-phase species.

Loinard et al. (2002) reported the observation of $\mathrm{D}_{2} \mathrm{CO} / \mathrm{H}_{2} \mathrm{CO}$ in two (SVS12 and SVS13) out of the four sources studied here. In both sources the measured $\mathrm{D}_{2} \mathrm{CO} / \mathrm{H}_{2} \mathrm{CO}$ ratio is around $4 \%$, a value similar to that found also in the other two Class I sources studied by Loinard et al. (2002), and just a factor two or three smaller than the average value measured in Class 0 sources. Hence the case of SVS12 and SVS13 is likely representative of the formaldehyde deuteration in Class I sources, and more generally in embedded low-mass protostars, within a factor two or three. Note that the $\mathrm{HDCO} / \mathrm{H}_{2} \mathrm{CO}$ ratio in embedded protostars is even larger, $\sim 10 \%$, as observed in the Class 0 source IRAS 16293-2422 (Loinard et al. 2000; van Dishoeck et al. 1995). Not to mention the case of the deuterated methanol, which is about as abundant as the main isotope (Parise et al. 2002).

The present observations clearly show that in low-mass protostars the solid $\mathrm{HDO} / \mathrm{H}_{2} \mathrm{O}$ ratio is lower than about $2 \%$ $(3-\sigma)$, definitively lower than the observed fractionation of the gas-phase formaldehyde. This is a crucial step towards the understanding of the deuteration from an observational point of view.

Before discussing the consequences of the comparison of these ice observations with gas-phase observations, it is important to understand where these ices are located along the line of sight and whether the ices we are probing have experienced the same chemical history as the ices that evaporated in the hot core and released the high-deuterated molecules such as formaldehyde and methanol into the gas-phase. In principle, a substantial part of the ice absorption may arise in the foreground quiescent gas not directly associated with the protostar itself (Boogert et al. 2002b). Observation of the pure solid $\mathrm{CO}$ band at $4.67 \mu \mathrm{m}$ may help elucidate how much thermal processing the ices have undergone and hence their proximity to the YSO. Indeed, pure CO absorption will only be present for ices less than $15 \mathrm{~K}$ and hence is expected to arise only in

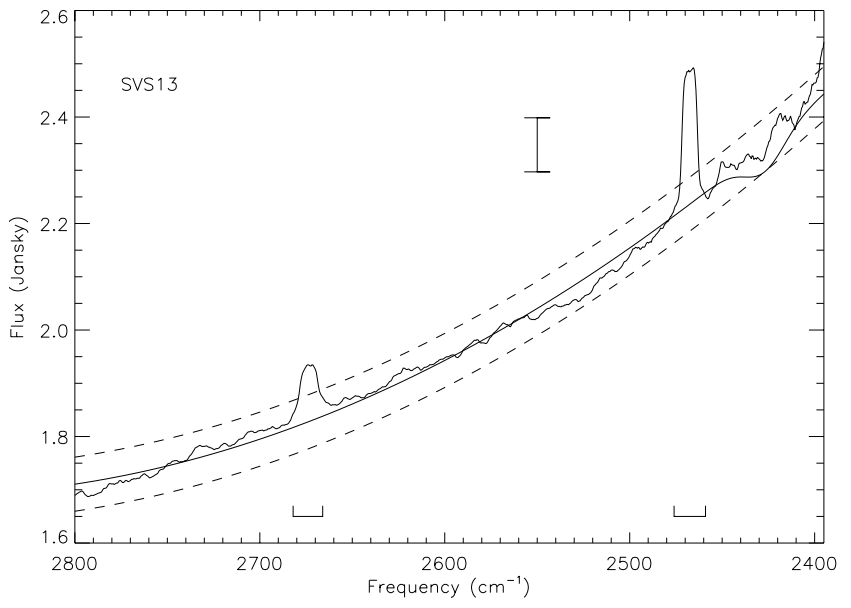

Fig. 6. OD stretch band for SVS13. The horizontal bars show the frequency ranges ignored in the continuum fit. The vertical bar is a representative $\pm 3 \sigma$ error bar. The maximum Gaussian absorption, superimposed as a solid line, has been computed under the assumption of crystalline ice (see text).

the dark foreground cloud. The CO band towards L1489 IRS has been observed and modelled by Boogert et al. (2002a). They find an optical depth of 0.4 for the pure CO component (see their Fig. 5). The distribution of pure solid CO in the Taurus molecular cloud has been well studied (Whittet et al. 1989; Chiar et al. 1995). The optical depth in the unperturbed dark cloud scales linearly with the total extinction $\left(\tau_{\text {pureCO }}=\right.$ $0.08\left(A_{\mathrm{v}}-6\right)$, Chiar et al. 1995). The pure CO optical depth towards L1489 IRS corresponds then to a visual extinction of 11 for the foreground cloud and a visual extinction of 18 for the envelope associated with the protostar. The $\mathrm{H}_{2} \mathrm{O}$ ice optical depth in the Taurus dark cloud shows a similar linear correlation with $A_{\mathrm{v}}\left(\tau_{\mathrm{H}_{2} \mathrm{O}}=0.08\left(A_{\mathrm{v}}-3\right)\right.$, Chiar et al. 1995). Hence, the $\mathrm{H}_{2} \mathrm{O}$ optical depth associated with the foreground cloud is only 0.6 and ice grain in the envelop of L1489 IRS contribute to $\tau_{\mathrm{H}_{2} \mathrm{O} \text {,ice }}=2.4$. Thus, our upper limit on the fractionation of $\mathrm{H}_{2} \mathrm{O}$ ice in the immediate environment of this protostar is $1 \%$.

In the following we discuss the consequences of these two sets of observations, gas-phase and solid-state deuteration. We start discussing two possibilities: either formaldehyde (and methanol) forms at the same time as water ices or water ices are formed in a previous phase and therefore they are imprinted with the - different - deuteration degree of this phase.

There is now a general consensus, supported by several observational and theoretical works, that both formaldehyde and methanol form on the grain surfaces, probably after hydrogenation of $\mathrm{CO}$ molecules that have stuck onto the grains (e.g. theoretical studies of Shalabiea \& Greenberg 1994; Tielens \& Whittet 1997; Charnley et al. 1997, and laboratory experiment of Watanabe \& Kouchi 2002), although one laboratory experiment seems to challenge this last hypothesis (Hiraoka et al. 2002). If we assume now that water ices are formed at the same time by hydrogenation of oxygen atoms sticking on the grain surfaces, the deuteration degree is set by the atomic $\mathrm{D} / \mathrm{H}$ ratio of the accreting gas (e.g. Tielens 1983; Caselli et al. 2002), and cannot be substantially different in the three species (water, formaldehyde and methanol), unless 
selective deuteration is at work in formaldehyde and methanol with respect to water. Detailed chemical models have been developed for the deuterium fractionation on grain surfaces. These lead to enhanced fractionation of formaldehyde with respect to that in other ice species centering on $\mathrm{H}$-abstraction reactions, driven by the zero-point energy difference (Tielens 1983). However, our insights in grain surface chemistry have evolved considerably since then and these models have not been fully revisited.

In the second hypothesis, water, formaldehyde and methanol would be formed during different phases characterized by different physical conditions which then may lead to different levels of fractionation (cf., Dartois et al. 2003). In particular, if the methanol and formaldehyde are formed during the later stages of accretion - when most of the gas has accreted already -, their fractionation could be high (Roberts et al. 2003). Such a model would require that the physical conditions in the accreting gas which favor deuteration also favor formaldehyde and methanol formation but not water formation. This hypothesis encounters some problems as the high abundance of formaldehyde and methanol in hot cores is generally thought to reflect the evaporation of water-rich ices (Loinard et al. 2000; Ceccarelli et al. 2001; Schoier et al. 2002). Nevertheless, observation of abundant $\mathrm{D}_{2} \mathrm{CO}$ in the outer envelop of IRAS 16293 (Ceccarelli et al. 2001) provides some observational support for onion-like mantle structure, with deuterated species trapped in the CO-rich ices that evaporate around $15 \mathrm{~K}$. In this scheme gas-phase observation of deuterated molecules would trace the deuteration ratio in these CO-rich ices, whereas the solid-phase observation would trace the bulk of the $\mathrm{H} 2 \mathrm{O}$-ices, where deuteration may be less important.

Alternatively, water ices are formed by condensation of water, copiously formed in molecular shocks, occuring during the cloud phase (Bergin et al. 1999). Also in this case the $\mathrm{HDO} / \mathrm{H}_{2} \mathrm{O}$ ratio would be low, reflecting the high temperatures $(\sim 300 \mathrm{~K})$ at which water would have been formed. Within this scenario, formaldehyde and methanol are not shock-produced and hence show quite different (higher) deuterium fractionations characteristic of grain surface (or gas phase) formation at low temperatures during the preshock phase. Of course, shocks also have other chemical consequences - notably enhanced abundances of $\mathrm{SiO}$ and $\mathrm{SO}$ are considered to be common tracers of shocks (e.g., Codella et al. 2002; Schilke et al. 1997, and references therein). However, this pertains to gas phase species and, moreover, cannot address the deuteration issue at hand here.

None of these models seem to be fully developped and all seem to have some difficulty explaining the observations. Perhaps, it is time to reconsider models for the selective deuteration of formaldehyde and methanol on grain surfaces.

\section{Conclusions}

We presented a search for solid HDO in grain mantles towards low-mass protostars. We did not detect HDO but derive upper limits on the $\mathrm{HDO} / \mathrm{H}_{2} \mathrm{O}$ frationation ratio of 0.5 to $2 \%$. These upper limits definitely show that solid water is much less deuterated than other molecules observed in the gas phase, such as formaldehyde and methanol. The origin of these differences is not fully understood.

\section{References}

Bachiller, R., Guilloteau, S., Gueth, F., et al. 1998, A\&A, 339, 49

Bergin, E. A., Neufeld, D. A., \& Melnick, G. J. 1999, AJ, 510, L145

Boogert, A. C. A., Hogerheijde, M. R., \& Blake, G. A. 2002a, ApJ, 568, 761

Boogert, A. C. A., Hogerheijde, M. R., Ceccarelli, C., et al. 2002b, ApJ, 570, 708

Carr, J. S., \& Tokunaga, A. T. 1992, ApJ, 393, L67

Caselli, P., Hasegawa, T. I., \& Herbst, E. 1993, ApJ, 408, 548

Caselli, P., Stantcheva, T., Shalabiea, O., Shematovich, V., \& Herbst, E. 2002, Planet. Space Sci., 50, 1257

Ceccarelli, C., Castets, A., Loinard, L., Caux, E., \& Tielens, A. G. G. M. 1998, A\&A, 338, L43

Ceccarelli, C., Loinard, L., Castets, A., et al. 2001, A\&A, 372, 998

Cernis, K. 1990, ApSS, 166, 315

Charnley, S. B., Tielens, A. G. G. M., \& Millar, T. J. 1992, ApJ, 399, L71

Charnley, S. B., Tielens, A. G. G. M., \& Rodgers, S. D. 1997, ApJ, 482, L203

Chiar, J. E., Adamson, A. J., Kerr, T. H., \& Whittet, D. C. B. 1995, ApJ, 455, 234

Codella, C., Scappini, F., Bachiller, R., \& Benedettini, M. 2002, MNRAS, 331, 893

Cushing, M. C., Vacca, W. D., \& Rayner, J. T. 2003, PASP, submitted (available on http://irtfweb.ifa.hawaii.edu/Facility/spex/)

Dartois, E. 1998, Ph.D. Thesis, Université de Paris VI

Dartois, E., d'Hendecourt, L., Thi, W., Pontoppidan, K. M., \& van Dishoeck, E. F. 2002, A\&A, 394, 1057

Dartois, E., Thi, W.-F., Geballe, T. R., et al. 2003, A\&A, 399, 1009

Hiraoka, K., Sato, T., Sato, J., et al. 2002, ApJ, 577, 265

Hogerheijde, M. R., van Dishoeck, E. F., Blake, G. A., \& van Langevelde, H. J. 1998, ApJ, 502, 315

Hogerheijde, M. R., \& Sandell, G. 2000, ApJ, 534, 880

Hogerheijde, M. R. 2001, ApJ, 553, 618

Jacq, T., Walmsley, C. M., Mauersberger, R., et al. 1993, A\&A, 271, 276

Jennings, R. E., Cameron, D. H. M., Cudlip, W., \& Hirst, C. J. 1987, MNRAS, 226, 461

Knee, L. B. G., \& Sandell, G. 2000, A\&A, 361, 671

Lefloch, B., Castets, A., Cernicharo, J., \& Loinard, L. 1998a, ApJ, 504, 109

Loinard, L., Castets, A., Ceccarelli, C., et al. 2000, A\&A, 359, 1169

Loinard, L., Castets, A., Ceccarelli, C., et al. 2002, Planet. Space Sci., 50, 1205

Looney, L. W., Mundy, L. G., \& Welch, W. J. 2000, ApJ, 529, 477

Molinari, S., Liseau, R., \& Lorenzetti, D. 1993, A\&AS, 101, 59

Parise, B., Ceccarelli, C., Tielens, A. G. G. M., et al. 2002, A\&A, 393, L49

Parise, B., et al., in preparation

Rayner, J. T., Toomey, D. W., Onaka, P. M., et al. 2003, PASP, 115, 362

Roberts, H., Herbst, E., \& Millar, T. 2003, ApJ, 591, 41

Sandell, G., \& Knee, L. B. G. 2001, ApJ, 546, 49

Sato, S., Nagata, T., Tanaka, M., \& Yamamoto, T. 1990, ApJ, 359, 192

Shalabiea, O. M., \& Greenberg, J. M. 1994, A\&A, 290, 266

Schilke, P., Walmsley, C. M., Pineau des Forets, G., \& Flower, D. R. 1997, A\&A, 321, 293 
Schoier, F. L., Jorgensen, J. K., van Dischoeck, E. F., \& Blake, G. A. Turner, B. E. 1990, ApJ, 362, L29 2002, A\&A, 390, 1001

Stantcheva, T., \& Herbst, E. 2003, MNRAS, 340, 983

Turner, B. E. 2001, ApJS, 136, 579

Teixeira, T. C., Devlin, J. P., Buch, V., \& Emerson, J. P. 1999, A\&A,

Vacca, W. D., Cushing, M. C., \& Rayner, J. T. 2003, PASP, 115, 389 347, L19

Tielens, A. G. G. M. 1983, A\&A, 119, 177

Tielens, A. G. G. M., \& Whittet, D. C. B. 1996, in Molecules in astrophysics: probes and processes: abstract book, 1-5 July 1996, Leiden, The Netherlands, ed. E. F. van Dishoeck, IAU Symp., 178,45 1995, ApJ, 447, 760

Watanabe, N., \& Kouchi, A. 2002, ApJ, 571, L173

Watson, W. D. 1973, ApJ, 181, L129

Whittet, D. C. B., Adamson, A. J., Duley, W. W., Geballe, T. R., \& McFadzean, A. D. 1989, MNRAS, 241, 707 